\title{
DESENVOLVIMENTO RADICULAR E INFLUXO DE NITROGÊNIO EM DUAS CULTIVARES DE ARROZ1
}

\author{
MARCEL GIOVANNI COSTA FRANÇA ${ }^{2}$, ROBERTO OSCAR PEREYRA ROSSIELLO ${ }^{3}$, EVERALDO ZONTA ${ }^{4}$ \\ ADELSON PAULO ARAÚJO ${ }^{5}$ e FLÁVIA TOLEDO RAMOS ${ }^{2}$
}

\begin{abstract}
RESUMO - Foi conduzido um experimento para avaliar as diferenças genotípicas nas taxas de absorção de nitrogênio, associadas aos padrões de desenvolvimento radicular, em duas cultivares de arroz (Oryza sativa L.). As cultivares IAC 4440 e Comum Branco, adaptadas, respectivamente, ao cultivo sob irrigação e sequeiro, foram crescidas em solução nutritiva, efetuando-se sete coletas em intervalos de dez dias. Apesar das pequenas diferenças entre as cultivares na produção de massa seca radicular, IAC 4440 apresentou maior área radicular total, com raízes mais finas e maior área radicular específica, como conseqüência de um maior perfilhamento, associado a maiores teores de nitrogênio em folhas e colmos. Os influxos radiculares de nitrogênio foram significativamente superiores em Comum Branco entre 35 e 55 dias após plantio, estádio em que principiaram as diferenças nos padrões de desenvolvimento radicular das cultivares. O menor influxo de nitrogênio em IAC 4440 foi compensado pela maior produção de área radicular, resultando em taxas de acumulação de nitrogênio similares nas duas cultivares
\end{abstract}

Termos para indexação: análise de crescimento, área radicular, Oryza sativa.

\section{ROOT DEVELOPMENT AND NITROGEN INFLUX OF TWO RICE CULTIVARS}

\begin{abstract}
An experiment was carried out to evaluate the genotypical differences on rates of nitrogen absorption, as related to root developmental patterns, on two rice (Oryza sativa L.) cultivars. The cultivars IAC 4440 and Comum Branco, adapted to irrigated and upland tillage respectively, were grown in nutrient solution, when seven harvests were performed at ten days intervals. Regardless the narrow differences between cultivars on root dry mass production, IAC 4440 showed greater total root surface area, with finer roots and higher specific root area, as a consequence of a more intensive tillering, associated with higher nitrogen concentration in leaves and stems. Nitrogen influxes into roots were significantly higher in Comum Branco between 35 and 55 days after planting, when differences on root developmental patterns of cultivars have initiated. The lower nitrogen influx of IAC 4440 was compensated by its greater production of root area, resulting in similar nitrogen accumulation rates on both cultivars
\end{abstract}

Index terms: growth analysis, Oryza sativa, root area.

${ }^{1}$ Aceito para publicação em 23 de outubro de 1998.

${ }^{2}$ Eng. Agr., Estudante de Pós-graduação, Dep. de Solos, Universidade Federal Rural do Rio de Janeiro (UFRRJ), BR $465 \mathrm{~km} \mathrm{7,} \mathrm{CEP} \mathrm{23890-000} \mathrm{Seropédica,} \mathrm{RJ.}$

${ }^{3}$ Eng. Agr., Dr., Prof. Adjunto, Dep. de Solos, UFRRJ. Bolsista do CNPq. E-mail: ropr@ufrrj.br

${ }^{4}$ Eng. Agr., M.Sc. Prof. Assistente, Dep. de Solos, UFRRJ. Email: ezonta@ufrrj.br

${ }^{5}$ Eng. Agr., Dr., Prof. Adjunto, Dep. de Solos, UfRRJ. Email: aparaujo@ufrrj.br

\section{INTRODUÇÃO}

Alguns estudos têm mostrado a existência de diferenças genotípicas na eficiência de absorção de nitrogênio (N) em arroz (Oryza sativa L.) (Furlani et al., 1986; Ferraz Junior et al., 1997). Furlani et al. (1986) verificaram em solução nutritiva eficiências similares na absorção de $\mathrm{N}$ entre linhagens de arroz de sequeiro e irrigado, com base na quantidade de $\mathrm{N}$ acumulado. Ferraz Junior et al. (1997) observaram 
maior eficiência de absorção de $\mathrm{N}$ para produção de grãos em cultivares melhoradas, de irrigação ou sequeiro, em relação a um grupo de variedades locais de sequeiro, tradicionalmente cultivadas no Estado do Maranhão. Por outro lado, foi observada a existência de algumas variedades locais cuja eficiência de absorção não diferiu significativamente das cultivares melhoradas de melhor desempenho (IAC-47 e IAC-899).

Estudando parâmetros cinéticos de influxo de $\mathrm{NH}_{4}{ }^{+}$, Teo et al. (1992) não encontraram diferenças entre três cultivares de arroz irrigado, quando avaliadas após 60 dias de crescimento em solução nutritiva. Deve-se considerar, todavia, que a absorção de $\mathrm{N}$ não depende apenas dos parâmetros cinéticos, mas decorre da complexa interação entre esses parâmetros, o estádio de desenvolvimento do sistema radicular, a concentração e a forma iônica de $\mathrm{N}$ na interface raiz-solução (Nye \& Tinker, 1977; Jungk, 1991; Fernandes \& Rossiello, 1995). Particularmente, diferenças genéticas em geometria radicular e densidade de ramificação têm importante papel na capacidade adaptativa às flutuações temporais e espaciais de disponibilidade de nutrientes (Van Vuuren et al., 1996; Williams \& Yanai, 1996).

Como cultivares de arroz adaptadas à inundação possuem sistemas radiculares superficiais de hábito plagiotrópico, densamente ramificados, enquanto as de sequeiro apresentam, em geral, sistemas mais profundos, predominantemente ortogeotrópicos, com menor ramificação lateral (Harada \& Yamazaki, 1993; Morita \& Yamazaki, 1993), é possível que essas diferenças em desenvolvimento radicular possam afetar diferencialmente as taxas de acumulação ou os influxos radiculares de $\mathrm{N}$, nos dois tipos de cultivares.

Em atenção a esses aspectos, foi realizado um estudo na fase pré-floração, em dois genótipos de arroz contrastantes quanto às condições de cultivo, nível de melhoramento e produtividade, objetivando identificar diferenças genotípicas na morfologia radicular, taxa de acumulação de $\mathrm{N}$ e influxo radicular de $\mathrm{N}$.

\section{MATERIAL E MÉTODOS}

Foram estudadas duas cultivares de arroz com estádios de crescimento coincidentes mas com distinta adaptação ecofisiológica. IAC 4440 é uma cultivar do grupo moderno, adaptada ao cultivo sob irrigação, com porte baixo, folhas estreitas e eretas, elevado perfilhamento e alto potencial produtivo (Usberti Filho et al., 1986). Já Comum Branco é uma variedade local de sequeiro, coletada no Estado do Maranhão, é de porte alto, folhas largas e decumbentes, baixo perfilhamento e menor potencial produtivo, mas com alto teor de proteína no grão e tolerância ao alumínio (Coradin \& Fonseca, 1982; Mendonça, 1991). Sob as condições climáticas vigentes no experimento, essas cultivares apresentam ciclo de duração média. O estudo limitou-se até 85 dias após plantio (DAP), porque até essa fase dá-se a acumulação de mais de $85 \%$ do total de $\mathrm{N}$ absorvido por IAC 4440 e Comum Branco durante um ciclo de 135 dias (Zonta, 1996).

Sementes esterilizadas superficialmente foram postas a germinar em areia autoclavada. Aos dez DAP, grupos de quatro plântulas foram transferidas para vasos de plástico contendo 3,3 L da solução nutritiva descrita por Furlani et al. (1986), a meia força iônica. Aos 15 DAP, a solução foi trocada por uma de força iônica total, ajustada a pH 5,0 $\pm 0,2$, que continha $60 \mathrm{mg} \mathrm{L}^{-1}$ de $\mathrm{N}$, com relação $\mathrm{N}-\mathrm{NO}_{3}{ }^{-}$: $\mathrm{N}_{-} \mathrm{NH}_{4}{ }^{+}$de 4,0 . Aos 35 DAP foi efetuado o desbaste, deixando-se duas plantas por vaso, que aos 45 DAP foram transferidas para vasos contendo $12 \mathrm{~L} \mathrm{de}$ solução. O volume de água deionizada necessário para compensar perdas por transpiração foi adicionado diariamente. $\mathrm{O}$ experimento foi conduzido em casa de vegetação, onde a temperatura média do ar oscilou entre $31^{\circ} \mathrm{C}$ e $35^{\circ} \mathrm{C}$, e a densidade de fluxo fotossintético, medida às $12 \mathrm{~h}$, teve valor de $1.350 \pm 640 \mathrm{mmol} \mathrm{m}^{-2} \mathrm{~s}^{-1}$.

Foram realizadas sete coletas em intervalos de dez dias, entre os 25 e 85 DAP (aproximadamente duas semanas antes da floração). Por ocasião de cada coleta, o pH da solução foi mensurado, e a solução nutritiva dos vasos restantes trocada. Em cada coleta, as plantas foram separadas em raízes, lâminas foliares e colmos + bainhas. A área radicular superficial foi estimada pelo método fotoelétrico (Rossiello et al., 1995), com o medidor eletrônico LI-COR 3000. Dado o aumento exponencial da massa radicular, a medição direta da área radicular só foi possível nas duas primeiras amostragens. A partir dos $45 \mathrm{DAP}$, a área radicular foi obtida a partir de medições em subamostras com aproximadamente $20 \%$ do peso fresco total (Rossiello et al., 1995). Nas duas primeiras amostragens, o volume radicular foi considerado equivalente ao peso fresco (Nye \& Tinker, 1977), e nas coletas subseqüentes igual ao volume de água deslocado pelas raízes numa proveta (Carrigan \& Frey, 1980). O raio médio radicular foi estimado a partir dos valores de volume e área radicular (Rossiello et al., 1995), e a área radicular específica calculada como a razão entre a área e a massa seca radicular. 
O material de cada porção vegetal foi secado em estufa a $60^{\circ} \mathrm{C}$, e a massa seca de raízes, colmos + bainhas e lâminas foliares foi registrada, sendo o teor de $\mathrm{N}$ determinado pelo procedimento micro Kjeldahl (Nelson \& Sommers, 1973). Assumindo-se que a única fonte de $\mathrm{N}$ disponível às plantas foi a solução nutritiva, o $\mathrm{N}$ total acumulado na planta (o somatório do $\mathrm{N}$ acumulado em cada porção) foi considerado como correspondente ao $\mathrm{N}$ total absorvido pelo sistema radicular (Van Vuuren et al., 1996; Ferraz Junior et al., 1997).

A análise dos dados foi orientada inicialmente pela análise de variância, desenvolvida como um fatorial $2 \times 7$ (cultivar $\times$ época de coleta), com quatro repetições. As variâncias dos dados primários mostraram forte heterogeneidade entre as coletas; desta forma, os dados foram transformados em logaritmo natural $\left(\log _{\mathrm{e}}\right)$ previamente à análise (Araújo et al., 1996). Além disso, foram ajustadas as seguintes funções aos dados primários, por processo iterativo (programa SAEG, Fundação Artur Bernardes, UFV): exponenciais polinomiais de $2 \circ$ e de $3 \circ$ grau, Gompertz, e logística. Na seleção do modelo, foi considerado o valor do coeficiente de determinação $\left(\mathrm{R}^{2}\right)$, conjuntamente com a tendência de variação temporal dos caracteres mensurados, tal como avaliada em experimento em vasos com solo franco-arenoso (Zonta, 1996). Foi escolhido o modelo logístico para ajuste dos dados de número de perfilhos, massa seca e conteúdo de $\mathrm{N}$ totais, $\mathrm{e}$ a exponencial de $2^{\circ}$ grau para a área radicular.

A taxa de acumulação de $\mathrm{N}\left(\mathrm{TA}_{\mathrm{N}}\right)$ e o influxo radicular de $\mathrm{N}\left(\mathrm{I}_{\mathrm{N}}\right)$ foram calculados segundo as expressões:

$T A_{N N}=\frac{d U_{N V}}{d T} \quad\left(\mathrm{mmol} \mathrm{N} \mathrm{dia}^{-1}\right)$

$I_{N}=\frac{1}{A_{R}} \cdot \frac{d U_{N}}{d T}\left(\mathrm{mmol} \mathrm{N} \mathrm{m}{ }^{-2} \mathrm{raiz} \mathrm{dia}^{-1}\right)$

onde $\mathrm{U}_{\mathrm{N}}$ é o total de $\mathrm{N}$ acumulado na planta, $\mathrm{A}_{\mathrm{R}}$ a área radicular e $\mathrm{T}$ o tempo (em DAP). No método funcional de análise de crescimento, existem dificuldades no tratamento estatístico das curvas derivadas das variáveis primárias (eqs. 1 e 2). Já o método clássico de análise de crescimento permite a comparação das taxas por procedimentos estatísticos usuais, apesar da elevada variabilidade intrínseca em seu cálculo (Venus \& Causton, 1979). Sendo assim, o $\mathrm{I}_{\mathrm{N}}$ também foi estimado como valor médio entre intervalos entre coletas através da expressão (Nye \& Tinker, 1977):

$\overline{I_{N}}=\frac{U_{N 2}-U_{N 1}}{A_{R 2}-A_{R 1}} \cdot \frac{\log _{e} A_{R 2}-\log _{e} A_{R 1}}{T_{2}-T_{1}}\left(\mathrm{mmol} \mathrm{N} \mathrm{m}{ }^{-2}\right.$ raiz dia $\left.^{-1}\right)$

(eq. 3)

onde os sufixos 1 e 2 representam coletas sucessivas, e a barra sobre $\mathrm{I}_{\mathrm{N}}$ denota estimativa média. A aplicação da equação 3 exige uma relação linear entre $A_{R}$ e $U_{N}$, e regressões entre estas variáveis apresentaram os coeficientes:

Comum Branco: $\mathrm{U}_{\mathrm{N}}=1,39+49,12 \mathrm{~A}_{\mathrm{R}}, \mathrm{R}^{2}=0,998$ (eq. 4)

IAC 4440: $\mathrm{U}_{\mathrm{N}}=-0,66+42,15 \mathrm{~A}_{\mathrm{R}}, \mathrm{R}^{2}=0,978 \quad$ (eq. 5)

As pendentes dessas regressões diferiram significativamente $(\mathrm{P}<0,001$, teste $\mathrm{t})$. Como o delineamento experimental foi completamente casualizado, a comparação dos valores de influxo foi efetuada pelo método de Venus \& Causton (1979), que permite o cálculo da variância das taxas sem pareamento de plantas, comparando-se os valores de cada cultivar pelo teste $\mathrm{t}$.

\section{RESULTADOS E DISCUSSÃO}

\section{Desenvolvimento radicular}

As diferenças entre cultivares na produção de massa seca radicular não foram muito expressivas, sendo significativas apenas em duas épocas amostrais (35 e 45 DAP), com superioridade de IAC sobre Comum Branco (Tabela 1). Entretanto, variações em extensão e configuração do sistema radicular foram de maior expressão, e começaram a manifestar-se a partir dos $35 \mathrm{DAP}$, evidenciadas por diferenças significativas na área radicular entre as cultivares, com superioridade de IAC 4440 entre 35 e 75 DAP (Fig. 1). A variação na área radicular foi explicada por uma função exponencial polinomial, que apresentou máximos aos 81 e 84 DAP, para IAC 4440 e Comum Branco, respectivamente (Fig. 1). Em experimento onde essas cultivares foram crescidas em vasos com solo, foram observados valores máximos de área radicular aos 75 e 83 DAP para IAC 4440 e Comum Branco respectivamente (Zonta, 1996). Isto pode ser atribuído ao número total de eixos nodais, que atinge seu máximo no período entre a iniciação da panícula e a floração (Yamazaki \& Harada, 1982). Freqüentemente, tais raízes nodais completam sua elongação antes, ou pouco depois dessa época, dependendo do número de folhas no colmo principal, de tal forma que o crescimento de raízes laterais pode estender-se até o estádio de maturação (Morita \& Yamazaki, 1993).

A cultivar Comum Branco mostrou uma redução abrupta da área radicular específica até os 55 DAP, continuando a declinar mais lentamente depois, até estabilizar em torno de $0,075 \mathrm{~m}^{2} \mathrm{~g}^{-1}$ aos 75 DAP 
(Tabela 1). A cultivar IAC 4440 exibiu a mesma tendência de variação, mas com valores superiores a Comum Branco durante todo o período de crescimento (Tabela 1). De Willigen \& Van Noordwijk (1987) calcularam valores de área radicular específica entre 0,1 e $0,2 \mathrm{~m}^{2} \mathrm{~g}^{-1}$ relativamente a raízes com raio entre 0,1 e $0,3 \mathrm{~mm}$, portanto, dentro da faixa de valores observados (Tabela 1).

Existe uma correlação positiva entre número de colmos e número de raízes nodais, assim como uma correlação inversa entre número de raízes nodais e seu diâmetro (Morita \& Yamazaki, 1993). Embora não tenha sido feita a contagem do número de raízes nodais, é possível inferir uma produção muito maior dessas raízes em IAC 4440 do que em Comum Branco, em vista das diferenças entre as cultivares no número de perfilhos (Fig. 2). A cultivar Comum Branco teve um aumento quase linear, até os 65 DAP, quando produziu um máximo de 20 perfilhos, enquanto a IAC 4440 teve um período de perfilhamento mais extenso, produzindo aos 75 DAP um número três vezes maior de perfilhos (Fig. 2). Esse comportamento da cultivar adaptada ao alagamento, deveu-se à prevalência de condições apropriadas à expressão da sua capacidade de perfilhamento: baixa taxa de difusão de oxigênio na cultura hidropônica (Pradham et al., 1973), condições favoráveis de luz e temperatura, e teores foliares de $\mathrm{N}$ adequados à emergência e sobrevivência dos perfilhos (Tabela 2).
Harada \& Yamazaki (1993) observaram que a contribuição de raízes secundárias ao total do sistema radicular, na forma de número, comprimento ou área superficial de raízes, é da ordem de $85-90 \%$, enquanto no volume total essa contribuição é menor, da ordem de $30 \%$. Possivelmente, por essa razão, as diferenças entre IAC 4440 e Comum Branco manifestaram-se principalmente nos parâmetros indicativos de ramificação (área radicular total e área radicular específica), enquanto as diferenças em volume foram menos marcadas e tenderam a seguir as variações na massa seca radicular (Tabela 1). Isto sugere um menor diâmetro médio de raízes nodais em IAC 4440, que deve ter contribuído, juntamente com uma maior densidade de laterais, para menores valores de raio radicular nessa cultivar. Em Comum Branco, a resposta foi inversa, isto é, menor número de perfilhos, maior raio radicular e menor área radicular específica (Tabela 1 e Fig. 2).

Esses dados indicam que as cultivares reproduziram as características morfológicas típicas dos sistemas radiculares de cultivares de sequeiro e de inundação, durante as fases de desenvolvimento vegetativo e maturação do grão (Harada \& Yamazaki, 1993; Morita \& Yamazaki, 1993). Essas diferenças morfológicas foram também evidentes na arquitetura da parte aérea, avaliadas visualmente: a cultivar IAC 4440 apresentou um porte mais baixo, um maior número de colmos mais

TABELA 1. Parâmetros radiculares de duas cultivares de arroz crescidas em solução nutritiva, em sete épocas de coleta (médias de quatro repetições) ${ }^{1}$.

\begin{tabular}{|c|c|c|c|c|c|c|c|c|}
\hline \multirow[t]{2}{*}{$\begin{array}{c}\text { Dias após } \\
\text { plantio }\end{array}$} & \multicolumn{2}{|c|}{$\begin{array}{l}\text { Massa seca } \\
\text { radicular } \\
\left(\mathrm{g} \mathrm{planta}^{-1}\right)\end{array}$} & \multicolumn{2}{|c|}{$\begin{array}{c}\text { Volume } \\
\text { radicular } \\
\left(\mathrm{cm}^{3} \text { planta }^{-1}\right) \\
\end{array}$} & \multicolumn{2}{|c|}{$\begin{array}{c}\text { Área radicular } \\
\text { específica } \\
\left(\mathrm{m}^{2} \mathrm{~g}^{-1}\right)\end{array}$} & \multicolumn{2}{|c|}{$\begin{array}{l}\text { Raio médio } \\
\text { radicular } \\
(\mathrm{mm})\end{array}$} \\
\hline & $\begin{array}{c}\text { Comum } \\
\text { Branco } \\
\end{array}$ & $\begin{array}{l}\text { IAC } \\
4440 \\
\end{array}$ & $\begin{array}{c}\text { Comum } \\
\text { Branco } \\
\end{array}$ & $\begin{array}{l}\text { IAC } \\
4440 \\
\end{array}$ & $\begin{array}{c}\text { Comum } \\
\text { Branco } \\
\end{array}$ & $\begin{array}{l}\text { IAC } \\
4440 \\
\end{array}$ & $\begin{array}{c}\text { Comum } \\
\text { Branco }\end{array}$ & $\begin{array}{l}\text { IAC } \\
4440 \\
\end{array}$ \\
\hline 25 & $0,05 \mathrm{a}$ & $0,04 \mathrm{a}$ & $0,5 \mathrm{a}$ & $0,5 \mathrm{a}$ & $0,161 b$ & $0,167 \mathrm{a}$ & $0,140 \mathrm{a}$ & $0,136 a$ \\
\hline 35 & $0,32 b$ & $0,46 \mathrm{a}$ & $4,6 b$ & $7,0 \mathrm{a}$ & $0,133 b$ & $0,140 \mathrm{a}$ & $0,218 \mathrm{a}$ & $0,217 \mathrm{a}$ \\
\hline 45 & $1,67 b$ & $2,09 \mathrm{a}$ & $15,7 \mathrm{~b}$ & $21,7 \mathrm{a}$ & $0,105 b$ & $0,128 \mathrm{a}$ & $0,228 \mathrm{a}$ & $0,162 b$ \\
\hline 55 & $5,57 \mathrm{a}$ & $5,69 \mathrm{a}$ & $47,0 \mathrm{a}$ & $53,9 \mathrm{a}$ & $0,084 b$ & $0,111 \mathrm{a}$ & $0,202 \mathrm{a}$ & $0,178 b$ \\
\hline 65 & $9,98 \mathrm{a}$ & $10,15 \mathrm{a}$ & $85,9 b$ & $97,5 \mathrm{a}$ & $0,084 b$ & $0,107 \mathrm{a}$ & $0,205 \mathrm{a}$ & $0,177 b$ \\
\hline 75 & $17,42 \mathrm{a}$ & $16,07 \mathrm{a}$ & $119,0 \mathrm{a}$ & $122,0 \mathrm{a}$ & $0,075 b$ & $0,099 \mathrm{a}$ & $0,181 \mathrm{a}$ & $0,154 b$ \\
\hline 85 & $20,32 \mathrm{a}$ & $16,20 \mathrm{a}$ & $136,0 \mathrm{a}$ & $143,0 \mathrm{a}$ & $0,075 \mathrm{~b}$ & $0,095 \mathrm{a}$ & $0,178 \mathrm{a}$ & $0,171 \mathrm{a}$ \\
\hline
\end{tabular}

${ }^{1}$ Médias seguidas de letras diferentes, na mesma linha, diferem pelo teste $\mathrm{F}$ a $5 \%$ de probabilidade.

${ }^{2}$ Dados originais, que foram transformados em $\log _{\mathrm{e}}$ antes da análise de variância. 
finos, e folhas mais estreitas e eretas em relação a Comum Branco.

\section{Acumulação de N}

As concentrações de $\mathrm{N}$ nas lâminas foliares, colmos, bainhas e raízes das duas cultivares foram máximas aos $35 \mathrm{DAP}$, e declinaram gradativamente,

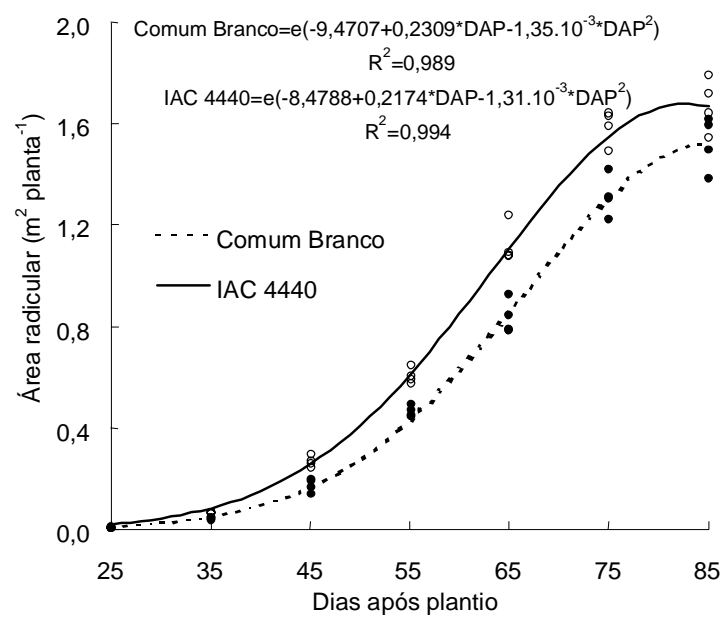

FIG. 1. Variação da área radicular em função do tempo de duas cultivares de arroz crescidas em solução nutritiva.

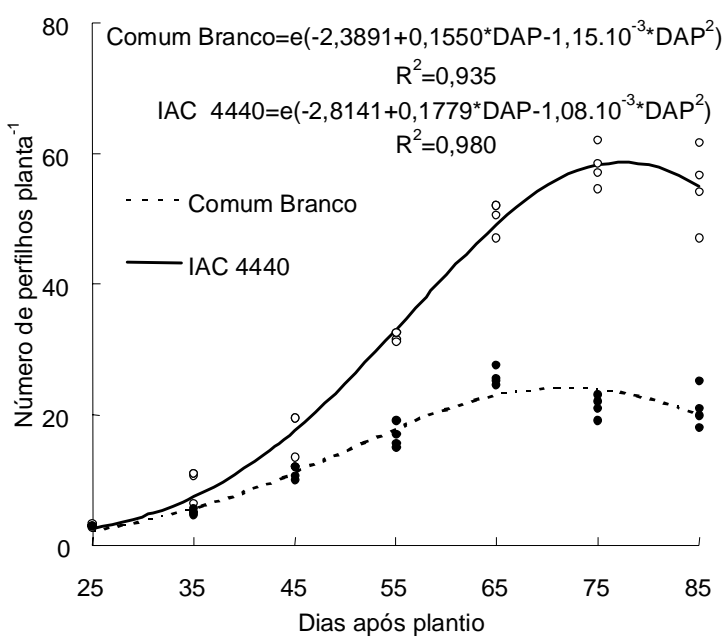

FIG. 2. Variação do número de perfilhos em função do tempo de duas cultivares de arroz crescidas em solução nutritiva. em função da idade (Tabela 2). Entre os 35 e 45 DAP Comum Branco apresentou maiores teores de $\mathrm{N}$ nas lâminas foliares, colmos, bainhas e raízes, mas após 55 DAP, IAC 4440 apresentou maiores teores de $\mathrm{N}$ nas lâminas foliares, colmos e bainhas, o que indica padrões diferenciados entre cultivares na utilização de $\mathrm{N}$ nas porções vegetativas. Valores acima de $30 \mathrm{mg} \mathrm{g}^{-1}$ nas lâminas foliares de IAC 4440 durante o período entre 35 e 75 DAP estão associados à elevada produção de perfilhos nessa cultivar. As concentrações de $\mathrm{N}$ em raízes não diferiram marcadamente, mas indicaram que até os 45 DAP, IAC 4440 usou mais eficientemente esse nutriente para produção de massa seca radicular (Tabela 2).

A cultivar IAC 4440 apresentou maior produção de matéria seca total aos $35 \mathrm{DAP}$, mas a partir de 75 DAP Comum Branco foi superior (Tabela 2), devido principalmente a uma maior alocação de biomassa em colmos e bainhas. Apesar desse padrão diferencial de partição de $\mathrm{Ce} \mathrm{N}$ entre órgãos vegetativos, a análise de variância indicou que não houve diferença significativa entre as cultivares no total de $\mathrm{N}$ absorvido, em nenhuma das épocas amostrais, e as curvas ajustadas para cada cultivar não diferiram (Fig. 3a). Em experimento em vasos com solo, o acúmulo de $\mathrm{N}$ nestas cultivares tendeu a estabilizar-se em torno de 15 dias após floração (Zonta, 1996). Silveira \& Machado (1990) também observaram que a quantidade total de $\mathrm{N}$ acumulada em IAC 4440 estacionou dez dias após floração, o que sugere a cessação do influxo radicular de $\mathrm{N}$. Desta forma, o melhor ajuste possível dos dados de acúmulo de $\mathrm{N}$ por causa do tempo foi uma função logística, em que o elevado valor de $\mathrm{R}^{2}$ justificou per se a escolha (Fig. 3a). A derivada da função ajustada fornece a taxa de acumulação de $\mathrm{N}$ (eq. 1), que atingiu valores máximos de 2,3 $\mathrm{mmol} \mathrm{dia}^{-1} \mathrm{em}$ torno de 64-67 DAP (Fig. 3b).

\section{Influxo radicular de $\mathbf{N}$}

Na Fig. 4 são apresentados os valores de influxo de $\mathrm{N}$, calculados segundo os métodos funcional (eq. 2) ou clássico (eq. 3) de análise de crescimento. Os influxos máximos, observados aos $25 \mathrm{DAP}$, por aplicação da equação 2 , da ordem de 12,3 e 
$7,4 \mathrm{mmol} \mathrm{N} \mathrm{m}{ }^{-2}$ dia $^{-1}$ para Comum Branco e IAC 4440, respectivamente (Fig. 4a), são menores que os valores calculados no tocante ao período 25-35 DAP (16,4 e $14,5 \mathrm{mmol} \mathrm{N} \mathrm{m}^{-2} \mathrm{dia}^{-1}$, Fig. 4b). Mais precisamente, calculando-se a média dos valores diários no período 25-35 DAP, a partir da Fig. 4a, obtiveram-se valores de 10,7 e $6,5 \mathrm{mmol} \mathrm{N} \mathrm{m}^{-2} \mathrm{dia}^{-1}$, o que indica que o método funcional subestimou em pelo menos $34 \%$ e $54 \%$ os influxos de Comum Branco e IAC 4440 nesse período. A razão para tal discrepância é que as funções ajustadas superestimaram a área radicular nesse período, e embora os valores absolutos sejam pequenos, o erro teve grande influência na estimativa do influxo. Quanto a períodos subseqüentes, as discrepâncias foram menores, e a partir dos 55 DAP ambas as estimativas são muito próximas, mostrando um declínio característico do influxo em função do tempo, tal como verificado em outras espécies (Waisel \& Eshel, 1991; Rossiello et al., 1995; Van Vuuren et al., 1996).

Em estudos de cinética de depleção de $\mathrm{NH}_{4}{ }^{+} \mathrm{em}$ duas cultivares de sequeiro, usando a mesma solução nutritiva e concentração de $\mathrm{N}$ que a do presente experimento, Baptista (1995) obteve estimativas de influxo entre 12 e $17 \mathrm{mmol} \mathrm{N} \mathrm{m}^{-2} \mathrm{dia}^{-1}$ aos $25 \mathrm{DAP}$, bem próximas às agora registradas. Cabe ressaltar que no presente caso a solução continha $\mathrm{NO}_{3}{ }^{-} \mathrm{e}$ $\mathrm{NH}_{4}{ }^{+}$, mas foi observada uma queda do $\mathrm{pH}$ da solução, a valores entre 4,0 e 4,5 ao final de cada período de amostragem. Essa tendência de acidificação foi verificada por Furlani et al. (1986), e é uma indicação indireta da absorção preferencial de $\mathrm{NH}_{4}{ }^{+}$sobre $\mathrm{NO}_{3}{ }^{-}$, característica em arroz (Fernandes \& Rossiello, 1995).

Calculando o influxo de $\mathrm{N}$ com base no peso fresco radicular para o período 25-35 DAP (eq. 3), foram obtidos valores de $0,15 \mathrm{e} 0,17 \mathrm{mmol} \mathrm{N} \mathrm{g}^{-1} \mathrm{dia}^{-1}$ referentes às cultivares IAC 4440 e Comum Branco, respectivamente. Esses resultados são similares aos observados por Kronzucker et al. (1998), entre 0,07 e $0,16 \mathrm{mmol} \mathrm{N} \mathrm{g}^{-1} \mathrm{dia}^{-1}$, e por Wang et al. (1993), de 0,06 a 0,30 $\mathrm{mmol} \mathrm{N} \mathrm{g}^{-1} \mathrm{dia}^{-1}$, em plantas de arroz com 21 dias em solução nutritiva com ${ }^{13} \mathrm{NH}_{4}{ }^{+}$. Tais comparações permitem concluir que a análise de crescimento fornece valores de influxo de $\mathrm{N}$ similares aos métodos da depleção da solução nutritiva ou uso de radioisótopos.

As dificuldades envolvidas na comparação estatística das curvas derivadas no método funcional de análise de crescimento não permitem afirmar que o influxo em Comum Branco foi significativamente maior que em IAC 4440 no início do período experimental (Fig. 4a), embora as diferenças significativas entre as pendentes das regressões $U_{N} \times A_{R}$ (eqs. 4 e 5) sugiram que este seja o caso. Já o método clássico de análise de crescimento (eq. 3) permite a comparação estatística das taxas, verifi-

TABELA 2. Massa seca total e teores de $\mathbf{N}$ nas porções vegetais em duas cultivares de arroz, crescidas em solução nutritiva, em sete épocas de coleta (médias de quatro repetições) ${ }^{1}$.

\begin{tabular}{|c|c|c|c|c|c|c|c|c|}
\hline \multirow[t]{2}{*}{$\begin{array}{l}\text { Dias após } \\
\text { plantio }\end{array}$} & \multicolumn{2}{|c|}{$\begin{array}{c}\text { Massa seca } \\
\text { total } \\
\left(\mathrm{g} \mathrm{planta}^{-1}\right) \\
\end{array}$} & \multicolumn{2}{|c|}{$\begin{array}{l}\text { Teor de } \mathrm{N} \text { nas } \\
\text { lâminas foliares } \\
\left(\mathrm{mg} \mathrm{g}^{-1}\right)\end{array}$} & \multicolumn{2}{|c|}{$\begin{array}{c}\text { Teor de } \mathrm{N} \text { nos } \\
\text { colmos e bainhas } \\
\left(\mathrm{mg} \mathrm{g}^{-1}\right)\end{array}$} & \multicolumn{2}{|c|}{$\begin{array}{c}\text { Teor de } \mathrm{N} \\
\text { nas raízes } \\
\left(\mathrm{mg} \mathrm{g}^{-1}\right)\end{array}$} \\
\hline & $\begin{array}{l}\text { Comum } \\
\text { Branco }\end{array}$ & $\begin{array}{l}\text { IAC } \\
4440\end{array}$ & $\begin{array}{c}\text { Comum } \\
\text { Branco }\end{array}$ & $\begin{array}{l}\text { IAC } \\
4440\end{array}$ & $\begin{array}{l}\text { Comum } \\
\text { Branco }\end{array}$ & $\begin{array}{l}\text { IAC } \\
4440\end{array}$ & $\begin{array}{c}\text { Comum } \\
\text { Branco }\end{array}$ & $\begin{array}{l}\text { IAC } \\
4440\end{array}$ \\
\hline 25 & $0,26 \mathrm{a}$ & $0,25 \mathrm{a}$ & $27,9 a$ & $29,3 \mathrm{a}$ & $18,7 \mathrm{a}$ & $19,2 \mathrm{a}$ & $13,1 \mathrm{a}$ & $11,4 \mathrm{a}$ \\
\hline 35 & $1,69 b$ & $2,16 \mathrm{a}$ & $46,1 \mathrm{a}$ & $43,1 \mathrm{~b}$ & $23,7 \mathrm{a}$ & $20,5 b$ & $21,3 \mathrm{a}$ & $15,6 \mathrm{~b}$ \\
\hline 45 & $7,74 \mathrm{a}$ & $8,74 a$ & $31,2 \mathrm{a}$ & $31,9 a$ & $13,0 \mathrm{a}$ & $11,6 b$ & $16,8 \mathrm{a}$ & $14,0 \mathrm{~b}$ \\
\hline 55 & $22,44 a$ & $21,05 \mathrm{a}$ & $26,3 b$ & $27,9 \mathrm{a}$ & $10,0 \mathrm{~b}$ & $11,1 \mathrm{a}$ & $7,1 \mathrm{a}$ & $6,5 \mathrm{a}$ \\
\hline 65 & $45,59 \mathrm{a}$ & $40,06 \mathrm{a}$ & $27,4 b$ & $30,5 \mathrm{a}$ & $9,3 \mathrm{~b}$ & $12,0 \mathrm{a}$ & $4,4 \mathrm{a}$ & $4,6 \mathrm{a}$ \\
\hline 75 & $73,64 a$ & $61,46 b$ & $27,8 \mathrm{~b}$ & $31,1 \mathrm{a}$ & $8,6 \mathrm{~b}$ & $10,9 a$ & $6,0 \mathrm{a}$ & $5,9 \mathrm{a}$ \\
\hline 85 & $107,60 \mathrm{a}$ & $93,47 \mathrm{a}$ & $22,7 b$ & $25,8 \mathrm{a}$ & $6,2 b$ & $7,8 \mathrm{a}$ & $6,4 \mathrm{a}$ & $6,9 a$ \\
\hline
\end{tabular}

${ }_{1}^{1}$ Médias seguidas de letras diferentes, na mesma linha, diferem pelo teste $\mathrm{F}$ a $5 \%$ de probabilidade; dados originais, que foram transformados em log antes da análise de variância. 
cando-se que o influxo em Comum Branco foi significativamente superior ao de IAC 4440 entre 35 e 55 DAP (Fig. 4b), estádio em que principiaram as diferenças entre cultivares nos padrões de desenvolvimento radicular (Fig. 1). Após 55 DAP, as diferenças não foram significativas, apesar de uma tendência de menores influxos em IAC 4440 (Fig. 4b).

A maior proliferação radicular em IAC 4440
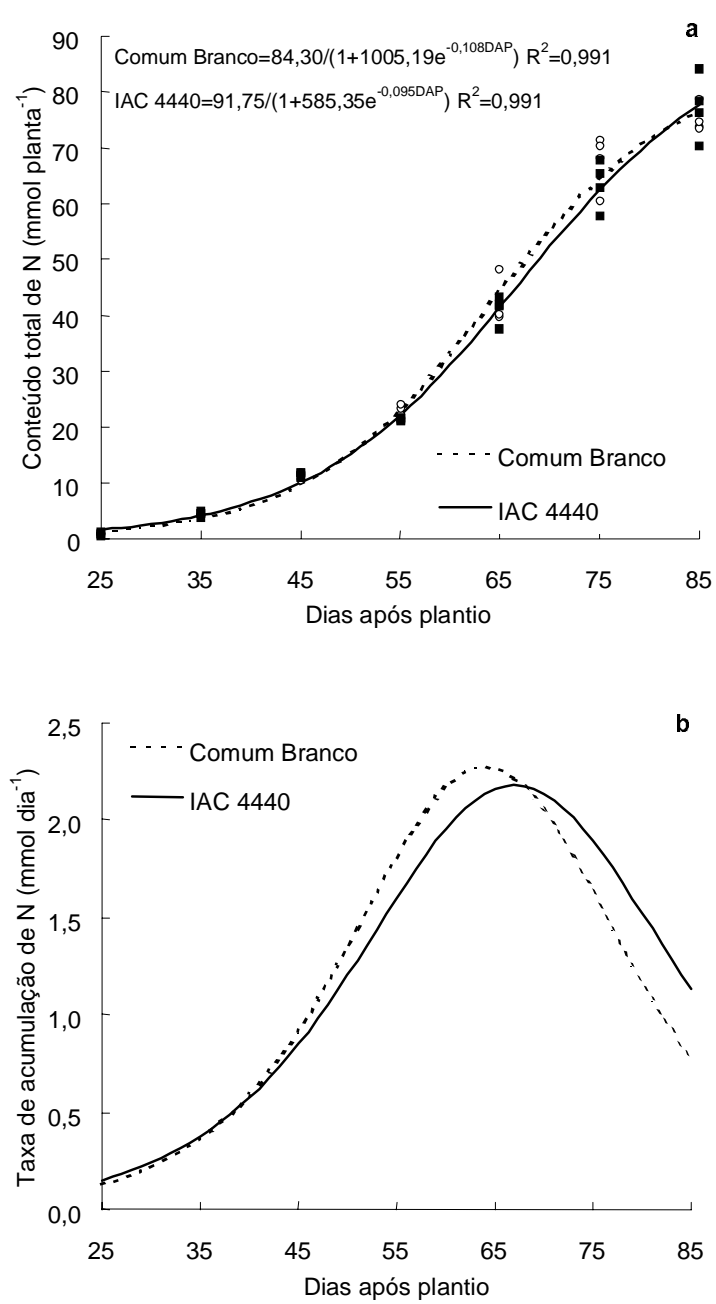

FIG. 3. Variação da acumulação de $\mathbf{N}$ em função do tempo de duas cultivares de arroz crescidas em solução nutritiva; a) $\mathrm{N}$ total acumulado; b) taxa de acumulação de $\mathbf{N}$.
(Fig. 1) implica redução da distância média entre os eixos radiculares, aumentando a competição entre raízes, uma vez que as zonas de depleção de $\mathrm{NO}_{3}{ }^{-} \mathrm{e}$ $\mathrm{NH}_{4}{ }^{+}$em torno delas tendem a sobrepor-se, reduzindo o influxo potencial em cada uma (Van Vuuren et al., 1996). Tal efeito contribuiu para anular
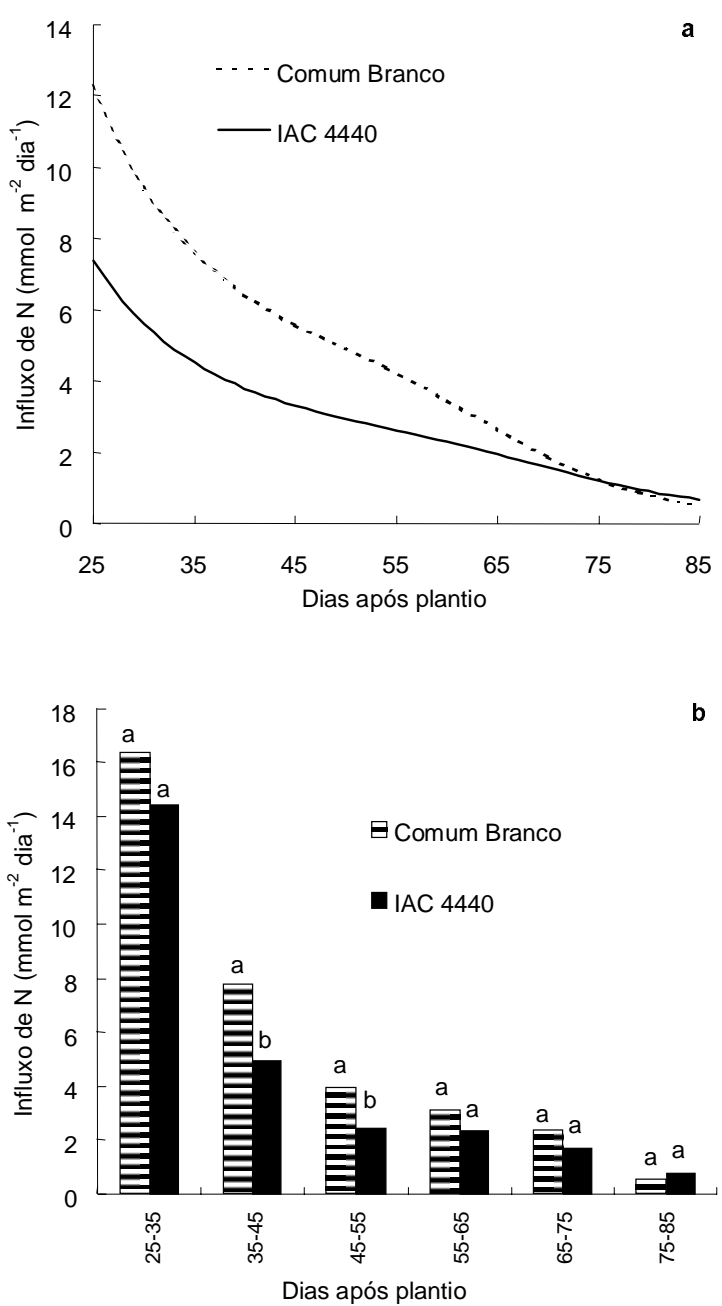

FIG. 4. Variação do influxo radicular de $\mathrm{N}$ em função do tempo de duas cultivares de arroz crescidas em solução nutritiva; a) valores instantâneos diários; b) valores médios para períodos amostrais de dez dias; médias sobrepostas por letras diferentes, dentro do mesmo período amostral, diferem pelo teste $t$ a $5 \%$ de probabilidade.

Pesq. agropec. bras., Brasília, v.34, n.10, p.1845-1853, out. 1999 
parcialmente a vantagem de um sistema radicular mais extenso e fino em IAC 4440, resultando em acúmulos de $\mathrm{N}$ similares nas duas cultivares (Fig. 3a). Em experimento em vasos com solo (Zonta, 1996), a produção de área radicular em IAC 4440 aos 80 DAP foi aproximadamente $60 \%$ menor do que a observada no presente experimento, e essa cultivar acumulou 12,8\% mais $\mathrm{N}$ que Comum Branco, com uma área radicular $8 \%$ menor. Isto sugere que a elevada produção de área radicular de IAC 4440 no presente experimento, em comparação com condições de solo, pode ter contribuído para uma redução de seu influxo radicular de $\mathrm{N}$.

$\mathrm{Na}$ aquisição de uma dada quantidade de nutriente, o influxo radicular numa espécie ou variedade pode ser baixo se, de forma compensatória, a extensão de seu sistema radicular é elevada (Jungk, 1991). Em uma cultivar como IAC 4440, com sistema radicular superficial e compacto, a taxa de acumulação de $\mathrm{N}$ pode ser controlada por uma capacidade inerentemente alta de regulação da sua densidade radicular (expressa, no presente caso, pelas variações em área radicular específica), e portanto um comparativamente baixo influxo não seria um problema em si. Já em Comum Branco, em que a pressão seletiva teria imposto um hábito radicular fortemente ortogeotrópico, com menor número de eixos nodais, mais grossos e alongados, e comparativamente menor proliferação lateral, um alto influxo é vantajoso. Com efeito, essa característica pode conferir uma capacidade de rápida absorção de acúmulos sazonais de $\mathrm{N}$ disponível no solo no início da estação de crescimento, competindo em melhor situação contra as perdas por lixiviação profunda. Contudo, Ferraz Junior et al. (1997) também tipificaram cultivares de sequeiro com baixa eficiência de absorção, o que mostra que os dados obtidos no presente trabalho não podem ser generalizados.

Os resultados aqui descritos parecem confirmar evidências prévias de diferenças relativamente estreitas na capacidade de absorção de $\mathrm{N}$ entre linhagens e cultivares de sequeiro ou inundação (Furlani et al., 1986), melhoradas ou não (Ferraz Junior et al., 1997), e com diferentes requerimentos de $\mathrm{N}$ (Teo et al., 1992). Contudo, a aparente similaridade nas taxas de absorção de $\mathrm{N}$ não implica potencial produtivo similar nas cultivares estudadas, uma ca- racterística dependente principalmente da capacidade genotípica de partição do $\mathrm{N}$ acumulado préfloração entre órgãos vegetativos e reprodutivos. Por outro lado, mecanismos compensatórios como os aqui discutidos devem ser considerados como componentes importantes na avaliação de diferenças genotípicas na absorção de $\mathrm{N}$; nesse sentido, o estudo da variação ontogenética do sistema radicular se faz necessário.

\section{CONCLUSÕES}

1. A cultivar IAC 4440, para irrigação, apresenta maior área radicular que a cultivar Comum Branco, de sequeiro, com raízes mais finas e maior área radicular específica.

2. Durante a fase juvenil de crescimento, o influxo radicular de $\mathrm{N}$ na cultivar Comum Branco é superior ao da cultivar IAC 4440.

3. As taxas de acumulação de $\mathrm{N}$ são similares nas duas cultivares, devido a um mecanismo compensatório entre influxo radicular de $\mathrm{N}$ e produção de área radicular.

\section{REFERÊNCIAS}

ARAÚJO, A.P.; ROSSIELLO, R.O.P.; SILVA, E.M.R da; ALMEIDA, D.L. de. Growth analysis of tomato colonized with arbuscular mycorrhizal fungi. Revista Brasileira de Ciência do Solo, Campinas, v.20, n.2, p.233-240, 1996

BAPTISTA, J.A. Cinética de absorção de $\mathbf{N H}_{4}^{+}$, efluxo de prótons e partição de $\mathbf{N}$ em arroz. Itaguaí: UFRRJ, 1995. 107p. Tese de Mestrado.

CARRIGAN, L.; FREY, K.J. Root volumes of Avena species. Crop Science, Madison, v.20, p.407-408, 1980.

CORADIN, L.; FONSECA, J.R. Coleta de germoplasma de arroz no Estado do Maranhão. Brasília: Embrapa-CENARGEN, 1982. 19p. (Documentos, 2).

DE WILLIGEN, P.; VAN NOORDWIJK, M. Roots, plant production and nutrient use efficiency. Wageningen: Agricultural Univ., 1987. 282p.

FERNANDES, M.S.; ROSSIELLO, R.O.P. Mineral nitrogen in plant physiology and plant nutrition. Critical Reviews in Plant Sciences, Boca Raton, v.14, p.111-148, 1995. 
FERRAZ JUNIOR, A.S.L.; SOUZA, S.R.; FERNANDES M.S.; ROSSIELLO, R.O.P. Eficiência do uso de nitrogênio para produção de grão e proteína por cultivares de arroz. Pesquisa Agropecuária Brasileira, Brasília, v.32, n.4, p.435-442, abr. 1997.

FURLANI, A.M.C.; BATAGLIA, O.C.; AZZINI, L.E Comportamento diferencial de linhagens de arroz na absorção e utilização de nitrogênio em solução nutritiva. Revista Brasileira de Ciência do Solo, Campinas, v.10, p.51-59, 1986.

HARADA, J.; YAMAZAKI, K. Morphology and development of roots. In: MATSUO, T.; HOSHIKAWA, K. (Eds.). Science of the rice plant. Morphology. Tokyo: Food and Agriculture Policy Research Center, 1993. v.1, p.133-161.

JUNGK, A.O. Dynamics of nutrient movement at the soil-root interface. In: WAISEL, Y.; ESHEL, A.; KAFKAFI, U. (Eds.). Plant roots: the hidden half New York: Marcel Dekker, 1991. p.455-481

KRONZUCKER, H.J.; KIRK, G.J.D.; SIDDIQI, M.Y.; GLASS, A.D.M. Effects of hypoxia on ${ }^{13} \mathrm{NH}_{4}^{+}$fluxes in rice roots. Plant Physiology, Rockville, v.116, p.581-587, 1998.

MENDONÇA, M.L.S. Estudo dos mecanismos de tolerância ao alumínio e sua variabilidade genotípica em arroz (Oryza sativa $\mathbf{L}$.). Itaguaí UFRRJ, 1991. 176p. Tese de Mestrado.

MORITA, S.; YAMAZAKI, K. Root system. In MATSUO, T.; HOSHIKAWA, K. (Eds.). Science of the rice plant. Morphology. Tokyo: Food and Agriculture Policy Research Center, 1993. v.1, p. 161-186

NELSON, D.W.; SOMMERS, L.E. Determination of total nitrogen in plant material. Agronomy Journal, Madison, v.65, p.109-112, 1973.

NYE, P.H.; TINKER, P.B. Solute movement in the soil-root system. Oxford: Blackwell, 1977. 342p.

PRADHAM, S.K.; VARADE, S.B.; KAR, S. Influence of soil water conditions on growth and root porosity of rice. Plant and Soil, The Hague, v.38, p.501-507, 1973

ROSSIELLO, R.O.P.; ARAÚJO, A.P.; MANZATTO, C.V.; FERNANDES, M.S. Comparação dos métodos fotoelétrico e da interseção na determinação de área, comprimento e raio médio radicular. Pesquisa Agropecuária Brasileira, Brasília, v.30, n.5, p.633-638, maio 1995
SILVEIRA, J.A.G.; MACHADO, E.C. Mobilização de nitrogênio e de carboidratos durante o desenvolvimento de panículas de duas cultivares de arroz. Revista Brasileira de Fisiologia Vegetal, Londrina, v.2, n.2, p.37-46, 1990.

TEO, Y.H.; BEYROUTY, C.A.; GBUR, E.E. Nitrogen, phosphorus, and potassium influx kinetic parameters of three rice cultivars. Journal of Plant Nutrition New York, v.15, n.4, p.435-444, 1992.

USBERTI FILHO, J.A.; AZZINI, L.E.; CAMARGO, O.B.A.; SOAVE, J.; SCHIMIDT, N.C.; VILELA, O.V.; GALLO, P.B.; SAKAI, M.; TISSELLI FILHO, O.; YANAI, K.; VITTI, P. IAC 4440: novo cultivar de arroz irrigado para o Estado de São Paulo. Campinas: IAC, 1986. 10p. (Boletim técnico, 107).

VAN VUUREN, M.M.I.; ROBINSON, D.; GRIFFTHS, B.S. Nutrient inflow and root proliferation during the exploitation of a temporally and spatially discrete source of nitrogen in soil. Plant and Soil, Dordrecht, v.178, p.185-192, 1996

VENUS, J.C.; CAUSTON, D.R. Plant growth analysis: a re-examination of the methods of calculation of relative growth and net assimilation rates without using fitted functions. Annals of Botany, London, v.43, p.633-638, 1979.

WAISEL, Y; ESHEL, A. Multiform behavior of various constituents of one root system. In: WAISEL, Y.; ESHEL, A.; KAFKAFI, U. (Eds.). Plant roots: the hidden half. New York: Marcel Dekker, 1991. p.39-52.

WANG, M.Y.; SIDDIQI, M.Y.; RUTH, T.J.; GLASS, A.D.M. Ammonium uptake by rice roots. II. Kinetics of ${ }^{13} \mathrm{NH}_{4}^{+}$influx across the plasmalemma. Plant Physiology, Rockville, v.103, 1259-1267, 1993

WILLIAMS, M.; YANAI, R.D. Multi-dimensional sensitivity analysis and ecological implications of a nutrient uptake model. Plant and Soil, Dordrecht, v.180, p.311-324, 1996

YAMAZAKI, K.; HARADA, J. The root system formation and its possible bearings on grain yield in rice plants. Japan Agricultural Research Quarterly, Tokyo, v.15, p.153-160, 1982.

ZONTA, E. Caracterização fisiológica da resposta à adubação nitrogenada em duas cultivares de arroz. Seropédica: UFRRJ, 1996. 164p. Tese de Mestrado. 